\title{
BMJ Open Description and prediction of outcome of drowning patients in New South Wales, Australia: protocol for a data linkage study
}

\author{
Amy E Peden (1) , ${ }^{1,2}$ Pooria Sarrami (1) ,3,4 Michael Dinh (D) ,3,5 \\ Christine Lassen (D) , ${ }^{3}$ Benjamin Hall, ${ }^{3}$ Hatem Alkhouri (D) , ${ }^{6,7}$ Lovana Daniel (D) , 4 \\ Brian Burns (iD) 5,8
}

To cite: Peden AE, Sarrami P, Dinh M, et al. Description and prediction of outcome of drowning patients in New South Wales, Australia: protocol for a data linkage study. BMJ Open 2021;11:e042489. doi:10.1136/ bmjopen-2020-042489

- Prepublication history and additional materials for this paper is available online. To view these files, please visit the journal online (http://dx.doi. org/10.1136/bmjopen-2020042489).

Received 06 July 2020 Revised 22 0ctober 2020 Accepted 27 November 2020

Check for updates

(c) Author(s) (or their employer(s)) 2021. Re-use permitted under CC BY-NC. No commercial re-use. See rights and permissions. Published by BMJ.

For numbered affiliations see end of article.

Correspondence to

Dr Amy E Peden;

a.peden@unsw.edu.au

\section{ABSTRACT}

Introduction Despite being a preventable cause of death, drowning is a global public health threat. Australia records an average of 288 unintentional drowning deaths per year; an estimated annual economic burden of $\$ 1.24$ billion AUD (\$2017). On average, a further 712 hospitalisations occur due to non-fatal drowning annually. The Australian state of New South Wales (NSW) is the most populous and accounts for $34 \%$ of the average fatal drowning burden.

This study aims to explore the demographics and outcome of patients who are admitted to hospitals for drowning in NSW and also investigates prediction of patients' outcome based on accessible data.

Methods and analysis This protocol describes a retrospective, cross-sectional data linkage study across secondary data sources for any person (adult or paediatric) who was transferred by NSW Ambulance services and/or admitted to a NSW hospital for fatal or non-fatal drowning between 1/1/2010 and 31/12/2019. The NSW Admitted Patient Data Collection will provide data on admitted patients' characteristics and provided care in NSW hospitals. In order to map patients' pathways of care, data will be linked with NSW Ambulance Data Collection and the NSW Emergency Department Data Collection. Finally patient's mortality will be assessed via linkage with NSW Mortality data, which is made up of the NSW Register of Births, Deaths and Marriages and a Cause of Death Unit Record File. Regression analyses will be used to identify predicting values of independent variables with study outcomes.

Ethics and dissemination This study has been approved by the NSW Population \& Health Services Research Ethics Committee. Results will be disseminated through peer-reviewed publications, mass media releases and at academic conferences. The study will provide outcome data for drowning patients across NSW and study results will provide data to deliver evidence-informed recommendations for improving patient care, including updating relevant guidelines.

\section{INTRODUCTION}

Drowning has been described as a threat to global public health, ${ }^{1}$ with recent estimates identifying 295,000 people die each

\section{Strengths and limitations of this study}

- This study explores the full extent of drowning, both on the basis of intent and mechanism, thus incorporating drowning cases less explored or not captured in mortality estimates.

- This study has limited outcome measures, lacking information about patient outcome beyond mortality and length of hospital stay.

- Findings will provide greater insight into pathways of care and clinical outcomes for drowning patients, resulting in evidence-informed recommendations for improving patient care, including updating relevant guidelines.

year around the world due to unintentional drowning. ${ }^{2}$ The fatal unintentional drowning toll is thought to be up to $40-50 \%$ higher than this estimate in developed nations (and even higher in developing countries) due to the additional impact of disaster and transportation-related drownings. ${ }^{34}$

Drowning may also be intentional in nature (ie, self-harm, homicide, infanticide) or have undetermined intent. Studies of self-harm-related drowning indicate it accounts for 2-3\% of intentional self-harm deaths in Australia, however significantly less research has been conducted on intentional drowning when compared with unintentional drowning. ${ }^{5}$

Drowning is defined as the process of experiencing respiratory impairment from submersion or immersion in liquid, with outcomes classified as death, morbidity and no morbidity. ${ }^{6}$ When compared with fatal drowning, non-fatal drowning is less understood, including global burden, characteristics, severity and long-term outcome. ${ }^{78}$

Drowning is a preventable cause of mortality and morbidity. Drowning occurs in a range of 
aquatic locations, in all seasons and in both metropolitan and rural locations. In Australia, an average of 288 unintentional fatal drownings are recorded annually, ${ }^{9}$ attracting an estimated economic burden of \$1.24billion each year (using 2017 metrics). ${ }^{10}$ Non-fatal drowning is also a considerable health hazard in Australia, warranting further attention, with an average of 712 hospital separations due to unintentional non-fatal drowning each year. ${ }^{9}$ When combined, there were a total of 1000 unintentional drowning incidents in Australia in 2018/2019, which represents a crude rate of 4.53 per 100,000 population. ${ }^{9}$ Intentional drowning data for Australia indicate a crude mortality rate of 0.23 per 100,000 between 2006 and 2014, lower than the crude rates for unintentional drowning (0.89), water transport-related drowning (0.17) and fatal drowning due to undetermined intent (0.09) during the same period. ${ }^{11}$

As the most populous state or territory in Australia, New South Wales (NSW) averages 99 unintentional drowning deaths, representing $34 \%$ of the national toll. ${ }^{9}$ In addition to this, NSW averages 155 unintentional non-fatal drownings requiring hospitalisation. ${ }^{7}$ Drowning therefore poses a significant burden to the health system in NSW.

Unintentional drowning disproportionately impacts children and young people with over half of the global burden occurring in those aged under 25 years. ${ }^{1}$ In Australia, children under the age of five record the highest rate of fatal and non-fatal unintentional drowning requiring hospitalisation, ${ }^{12}$ and unintentional drowning is the third leading cause of premature death amongst children aged 1-14 years in Australia. ${ }^{13}$

The treatment of drowning has been identified as one of the most costly forms of paediatric trauma treatment ${ }^{14}$ and drowning has been identified as having a higher mortality risk within 30 days of hospitalisation. ${ }^{15}$ For effective evaluation and treatment of drowning patients, it is recommended that patients will be transferred to an Emergency Department (ED) ${ }^{16}$ The main priority in the treatment of drowning is reversing hypoxemia by enabling adequate oxygenation and ventilation via oxygen therapy and in some cases via non-invasive or invasive mechanical ventilation. ${ }^{17}$ Other major priorities include hypothermia management, neuroprotective strategies in comatose patients and post-ROSC (return of spontaneous circulation) cardiac arrest care. By exploring pathways of care for drowning patients, gaps in the literature, particularly around severity and outcome of non-fatal drowning requiring hospitalisation can be addressed and evidenceinformed recommendations for improving patient care can be made.

Data linkage studies exploring unintentional drowning have previously been conducted in Queensland (with a focus on children and adolescents) ${ }^{18}$ and in Victoria (exploring fatal and non-fatal drowning cases attended by ambulance) ${ }^{19}$ A previous study in NSW explored patients 16 years and older with a view to identify the impact of exposure on fatal and non-fatal drowning risk, ${ }^{20}$ however no prior study has explored both paediatric and adult drowning cohorts in NSW across the whole spectrum of treatment. Therefore, utilising data linkage, this study aims to explore the outcome of patients who are admitted to hospitals for drowning in NSW and also investigate prediction of patients' outcome based on the data available at the time of presentation to ED. This article describes the protocol for the study. Future updates on the project can be found on the NSW Institute of Trauma and Injury Management's website (https://www. aci.health.nsw.gov.au/get-involved/institute-of-traumaand-injury-management/research/itim-involvement-intrauma-research/current-research-projects).

\section{METHOD}

This is a retrospective, cross-sectional data linkage study of drowning (unintentional, intentional and undetermined intent) in NSW, Australia. Data will be collated for any person (adult and paediatric) who was transferred by NSW Ambulance services and/or admitted to a public or private hospital in NSW for a fatal or non-fatal drowning between 1/1/2010 and 31/12/2019. Although 'non-fatal drowning' is the accepted terminology with respect to a drowning incident where the person does not die, ${ }^{6}$ the term 'near-drowning' is used in this protocol where it refers to existing system-based codes for the capture of non-fatal drowning patients.

\section{Data sources and collection}

This study involves secondary data collection from data sources across a patient's pathway of care, including prehospital and hospital, through to death for fatal cases. The NSW Admitted Patient Data Collection (APDC) will provide data on the characteristics and the provided care for the patients admitted to hospitals in NSW for fatal or non-fatal drowning experiences. The APDC has been described elsewhere ${ }^{20}$ but in brief, it includes information on inpatient separations from NSW public or private hospitals, private day procedures and public psychiatric hospitals. It includes information on episodes of care in hospital that end with discharge, transfer or death of the patient, or when the service category for the patient changes. Although hospitalisations of NSW residents which occurred in another Australian state or territory are included, such cases will not be included in this study due to difficulties regarding linkage across other NSWbased data sources.

In order to map patients' journey, APDC data will be linked with NSW Ambulance Data Collection. In addition, data will be linked to NSW Emergency Department Data Collection (EDDC) to record the care patients might have received in the ED. Finally patient's mortality will be assessed via data linkage with NSW Mortality data, which is made up of the NSW Register of Births, Deaths and Marriages and a Cause of Death Unit Record File (COD URF) (figure 1).

Both APDC and NSW Ambulance Data will be used to define the cohort. This approach means that the 


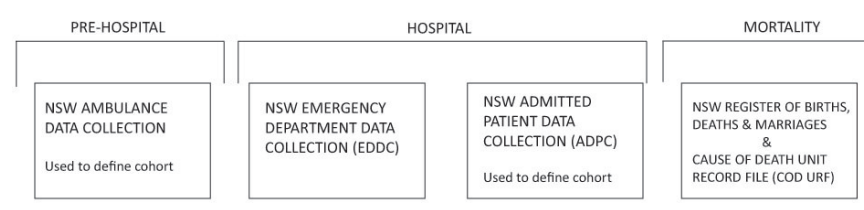

Figure 1 Flow chart depicting study data sources across the pathway of care.

hospital data will have good accuracy in identification (though limited to cases that are admitted to hospital) while ambulance data (though less precise) will also allow identification of those cases not admitted to hospital. Variables requested from the databases and their purpose are outlined in online supplemental table 1 . With respect to the identification of drowning cases through the NSW Ambulance databases, the data elements outlined in table 1 will be used.

To determine mortality within hospital cases, International Classification of Diseases (ICD) codes will be used. The codes in table 2 cover both principal diagnosis and external cause codes from both ICD-9 and ICD-10. Registry of Births, Deaths and Marriages (RBDM) and CODURF data are linked to ADPC and drowning cases are found in ADPC or the Ambulance data to identify deaths after discharge from hospitals.

Variables collected from the NSW ADPC and NSW EDDC will include predictor variables (eg, age) and outcome variables (eg, episode leave days total). The NSW Ambulance Data Collection variables are obtained to analyse the patient transfer pathways. Readmissions will be identified via mode of arrival (emergency, readmission within 30 days of index admission). Injury profiles will be described based on injury codes (coded as Abbreviated Injury Scale codes) and Injury Severity Score.

Finally, this database will provide outcomes (including length of stay in hospital, Intensive Care Unit (ICU) length of stay and neurological outcome measures such as Glasgow Coma Score (GCS) or return of spontaneous circulation (ROSC)). Date of death which will indicate mortality, as an outcome, will be obtained via the RBDM containing mortality information for deaths occurring in NSW. Cause of death will also be assessed by Cause of Death Unit Record File. Length and duration of transfer will be calculated based on data obtained via ED and NSW Ambulance Data collections.

Table 1 Data elements in the NSW ambulance databases

\begin{tabular}{ll}
\hline Name of datasets & $\begin{array}{l}\text { Coding/method for } \\
\text { identification and extraction }\end{array}$ \\
\hline $\begin{array}{l}\text { Paper-based Patient } \\
\text { Healthcare Record (PHCR) }\end{array}$ & Protocol1-Protocol8: drowning \\
$\begin{array}{ll}\text { Electronic medical record } \\
\text { (eMR) }\end{array}$ & $\begin{array}{l}\text { 'Protocols': drowning } \\
\text { Case nature: drowning/ } \\
\text { immersion }\end{array}$ \\
$\begin{array}{ll}\text { Computer-aided dispatch } \\
\text { (CAD) }\end{array}$ & $\begin{array}{l}\text { Problem: drowning (near)/diving/ } \\
\text { scuba }\end{array}$ \\
\hline
\end{tabular}

\section{Data linkage and security}

Data linkage will be performed by the Centre for Health Record Linkage (CHeReL) in accordance with standard models. ${ }^{21-23}$ In brief, once approvals have been granted, data from different databases are provided to CHeReL. Records from each dataset are split into two separate files (one containing identifier information such as name and address and the other containing content information (in this case information on the drowning incident and treatment provided)). Identifier data is used by CHeReL's Data Linkage Unit using a combination of matching methods to identify and distinguish between individuals. Identified data belonging to each individual is assigned an arbitrary Person Number, which replaces the identifier data.

Using an encrypted version of the arbitrary Person Number, CHeReL's Data Integration Unit then makes a research Project-specific Person Number (PPN) for each individual. This is then joined to the relevant data from the datasets once approved. All records for an individual in any approved project dataset will have the PPN. Approved data is then released to the researcher. Using the PPN, the researcher can combine records for an individual without accessing information about their identity. ${ }^{23}$

The master file containing identifying information and master linkage key will be stored in a secure network partition at CHeReL. Files will be transferred electronically using file encryption. Only a de-identified version of the dataset will be used for analysis by study investigators. All analyses will be undertaken on site within Agency for Clinical Innovation (ACI) offices using standard statistical software. Data will be recorded on secure local network related to NSW HealthShare accessible only by authorised members. All members of the research team will access aggregated data only and data will not be transferred to any other locations out of NSW Institute of Trauma and Injury Management (ITIM). Files will be kept in password-protected computers at the ACI for the duration of the study and 7 years following completion of the project, after which time they will be deleted.

There is a small chance of re-identification of data. In order to minimise the risk, the researchers, who can access only data with identifiers removed, will store the potentially re-identifiable data in secure locations at the ACI. Re-identification of data will be possible only by CHeReL or the data custodians, who also follow strict measures to protect data security and privacy. In comparison to the risk of re-identification of data (which is very unlikely), this study will contribute to improving data outcomes for drowning and neardrowning patients across NSW and providing better models of care for those patients.

Identifiers will be removed from the dataset provided to the research team. Data will include data on patients who have a drowning experience and will assist to address the following queries (table 3 ). 


\begin{tabular}{|c|c|c|c|}
\hline ICD iteration & Section & Codes & Explanation \\
\hline \multicolumn{4}{|l|}{ ICD 9} \\
\hline & E910 & & Accidental drowning and submersion \\
\hline & & E910.0 & While water skiing \\
\hline & & E910.1 & While engaged in other sport or recreational activity with diving equipment \\
\hline & & E910.2 & While engaged in other sport or recreational activity without diving equipment \\
\hline & & E910.3 & While swimming or diving for purposes other than recreation or sport \\
\hline & & E910.4 & In bathtub \\
\hline & & E910.8 & Other \\
\hline & & E910.9 & Unspecified place of occurrence code \\
\hline \multicolumn{4}{|l|}{ ICD10 } \\
\hline & W65-W74 & & Drowning and submersion \\
\hline & & W65 & While in bathtub \\
\hline & & W66 & Following fall into bathtub \\
\hline & & W67 & While in swimming pool \\
\hline & & W68 & Following fall into swimming pool \\
\hline & & W69 & While in natural water \\
\hline & & W70 & Following fall into natural water \\
\hline & & W73 & Other specified \\
\hline & & W74 & Unspecified \\
\hline & & T751 & Drowning and non-fatal submersion (often used a diagnosis code) \\
\hline & & V90 & Drowning and submersion due to accident to watercraft \\
\hline & & V92 & $\begin{array}{l}\text { Drowning and submersion due to accident on board watercraft, without accident to } \\
\text { watercraft }\end{array}$ \\
\hline & & X38 & Victim of flood \\
\hline & & Y21 & Drowning and submersion, undetermined intent \\
\hline & & $\mathrm{X} 71$ & Intentional self-harm by drowning and submersion \\
\hline & & X92 & Assault by drowning and submersion \\
\hline
\end{tabular}

\section{Analysis}

Analysis will explore the following outcomes of interest:

1. Hospital length of stay (days) - prolonged length of stay will be defined as length of stay greater than 30 days.

\section{Table 3 Data fields used to address queries}

\section{Description of the cohort}

\section{Demographic information}

2 Patients' pathways (where they have been picked up and received care)

\section{Hospital costs}

\section{Outcome and predicting variables}

1 Predictor variables (eg, demographic, vital signs on arrival, transfer time and distance, therapeutic interventions)

2 Patients' outcome (eg, mortality, hospital and ICU length of stay)
2. Re-admission to hospital within 30 days of discharge from index admission.

3. Discharge to rehabilitation in patients who survive to discharge.

4. Mortality.

5. Yearly count of patients, disaggregated by age and sex.

6. Hospital costs.

\section{Subgroups}

Any person who has had a drowning or non-fatal drowning experience and were admitted to a hospital in NSW between 1/1/2010 and 31/12/2019 will be included in the study. However, the following subgroups will be considered based on the aforementioned variables.

- Aged $<16$ years old.

- Aged $<5$ years old.

- Patients living in remote or very remote areas.

Statistical analysis

The present research seeks to understand the association of outcome of drowning patients (length of stay, readmission 
rates, hospital costs and mortality) with predictor variables (demographic, vital signs, transfer time and length and treatments). Analyses conducted using this study's data will be non-identifiable. Regression analyses will be used to identify predicting value of independent variables with study outcomes. The main analysis will be adjusted using measures of socioeconomic variables such as Socioeconomic Index for Advantage (SEIFA), demographic status and cultural background, and place of birth. Using the ADPC database's 'diagnosis codes', descriptive statistics will be used to plot and analyse trends in various comorbidities amongst specific subgroups of patients. The analysis will be performed using SAS software (version 9.4) ${ }^{24}$ The cost of hospital treatment is calculated using the Australian Refined Diagnosis Related Group (AR-DRGs). The AR-DRGs is an Australian admitted patient classification system which provides a clinically meaningful way of relating number and type of patients treated in a hospital to the resources required by the hospital. ${ }^{25}$

\section{Ethics approvals and issues of consent}

Ethics approval was granted by NSW Population \& Health Services Research Ethics Committee (approval number: HREC/18/CIPHS/19). It is impracticable to seek consent, because the research is retrospective based on data that is already collected from around 2000 patients who were admitted to hospital in the previous 10 years (2010-2019). Data received by researchers is de-identified.

\section{Patient and public involvement}

It will be impractical to identify and contact those patients as we will not have access to the identifiable data. Patients will not be required to actively participate. The data that the patients have already provided as part of their normal care will be analysed. In addition, patients' privacy will be protected as no one will contact patients and researchers will access data with identifiers removed. In addition, only unit record data will be analysed and reported which will not identify individual patients, therefore it will not be feasible to directly report back to the patients involved. However, study findings will be communicated to the public through mass media releases and to the scientific community via conference presentations and peer-reviewed publications. Study findings will be communicated to relevant wider patient communities through research partner Royal Life Saving Society Australia's state and territory member organisations who work with those who have experienced drowning. Findings will also be disseminated through NSW Ambulance and NSW Health to relevant stakeholders involved in the treatment of drowning patients.

\section{DISCUSSION}

The study will provide outcome data for drowning patients across NSW and also investigate prediction of patients' outcome based on accessible data such as demographic information, vital signs on arrival to hospital and length of transfer. It is anticipated, over the 10-year data period, that around 2000 patients will be identified through the APDC and NSW Ambulance data systems.

This study will explore temporal trends in drowning, quantify factors impacting drowning risk such as age, rurality (both drowning incident location and patient residential location) and SEIFA classification. Study findings will identify predictors impacting patient outcomes, factors impacting readmission and outcome of those discharged alive, as well as costs associated with treating drowning incidents in NSW, for both paediatric and adult patients.

Findings will contribute to producing better models of care for drowning patients across NSW, including influencing guidelines as appropriate. Improved models of care will contribute to reduction in mortality and morbidity and reduced burden on the health system. Cost savings on improvements in hospital-provided care for drowning patients are likely to be large. ${ }^{4}$ The result of this study will be available to public and scientific community via mass media releases, oral conference presentations and written publications in peer-reviewed scientific journals.

There are several strengths associated with this study. We define our population in two different ways to maximise our chance of capturing the drowning cases. This is done through the use of both pre-hospital and hospital data, allowing for more comprehensive capture rather than relying on one data source alone. Data linkage allows patients to be followed along their pathway of care, including identifying readmission and allowing for the exploration of severity among hospitalised non-fatal cases, an area so far under-explored. ${ }^{712}$ This study also explores the full extent of drowning, both on the basis of intent and mechanism, thus incorporating drowning cases less explored or not captured in mortality estimates. ${ }^{2-4}$ Similarly, while several data linkage studies have focused on children and adolescents, ${ }^{14} 18$ this study includes both paediatric and adult patients. The data is also longitudinal in nature, allowing for the exploration of temporal trends. The identification of drowning cases in ambulance datasets was difficult; this protocol provides a method for identifying these previously hard to extract cases. There are however, limitations associated with this study. Though an in-depth exploration of the issue, the findings represent care provided to drowning patients in one state only. It may be possible that some drowning cases may be missed in ambulance data if non-drowning descriptions are used. Drowning patients may also not be captured in this study if missing from Ambulance and hospital data (eg, if brought by private vehicle to the ED and then are discharged from ED without admission to hospital). Similarly, those patients who are found dead at the scene might not be captured in this data. This study has limited outcome measures, lacking information about patient outcome beyond mortality, length of hospital stay, readmission and discharge to rehabilitation. Exploring the association of inpatient care with long-term patient outcomes is another important area in future studies. 


\section{CONCLUSIONS}

Drowning is a preventable cause of death and injury with a high incidence in NSW representing a burden to the health system. This protocol outlines a study which aims to explore pathways of care for drowning victims from pre-hospital intervention, to care in hospital, to death in cases of fatal drowning. Findings will identify predictors of outcome as well as high-risk groups for the targeting of prevention efforts. Ultimately the results of the study will inform treatment guidelines, and thus improve outcomes for those hospitalised due to drowning.

\section{Author affiliations}

${ }^{1}$ School of Population Health, Faculty of Medicine, UNSW Sydney, Kensington, New South Wales, Australia

${ }^{2}$ Royal Life Saving Society Australia, Broadway, New South Wales, Australia ${ }^{3} \mathrm{NSW}$ Institute of Trauma and Injury Management, NSW Agency for Clinical Innovation, St Leonards, New South Wales, Australia

${ }^{4}$ South Western Sydney Clinical School, University of New South Wales, Liverpool, New South Wales, Australia

${ }^{5}$ The University of Sydney, Sydney Medical School, Sydney, New South Wales, Australia

${ }^{6}$ Emergency Care Institute, Agency for Clinical Innovation, St Leonards, New South Wales, Australia

${ }^{7}$ Faculty of Medicine, University of New South Wales, Sydney, New South Wales, Australia

${ }^{8}$ Greater Sydney Area Helicopter, Emergency Medical Service, NSW Ambulance, Sydney, New South Wales, Australia

\section{Twitter Amy E Peden @amyepeden}

Acknowledgements We thank the NSW Ministry of Health and the Centre for Health Record Linkage (CHeReL) for access and linkage of data. We also thank the in-kind support of the NSW Institute of Trauma and Injury Management and Royal Life Saving Society - Australia.

Contributors PS and MD conceptualised the study. PS, MD, CL and AEP designed the study. PS will conduct the data analysis, and MD and AEP will interpret the findings. AEP and PS drafted the manuscript with revisions provided by $\mathrm{MD}, \mathrm{CL}$, BH, HA, LD and BB. All authors reviewed and approved the final manuscript as submitted.

Funding This research is made possible by funding and in-kind support provided by the NSW Institute of Trauma and Injury Management. This research is supported by Royal Life Saving Society - Australia to aid in the prevention of drowning. Research at Royal Life Saving Society - Australia is supported by the Australian Government.

Competing interests None declared.

Patient consent for publication Not required.

Provenance and peer review Not commissioned; externally peer reviewed.

Supplemental material This content has been supplied by the author(s). It has not been vetted by BMJ Publishing Group Limited (BMJ) and may not have been peer-reviewed. Any opinions or recommendations discussed are solely those of the author(s) and are not endorsed by BMJ. BMJ disclaims all liability and responsibility arising from any reliance placed on the content. Where the content includes any translated material, BMJ does not warrant the accuracy and reliability of the translations (including but not limited to local regulations, clinical guidelines, terminology, drug names and drug dosages), and is not responsible for any error and/or omissions arising from translation and adaptation or otherwise.

Open access This is an open access article distributed in accordance with the Creative Commons Attribution Non Commercial (CC BY-NC 4.0) license, which permits others to distribute, remix, adapt, build upon this work non-commercially, and license their derivative works on different terms, provided the original work is properly cited, appropriate credit is given, any changes made indicated, and the use is non-commercial. See: http://creativecommons.org/licenses/by-nc/4.0/.

\section{ORCID iDs}

Amy E Peden http://orcid.org/0000-0002-6424-1511
Pooria Sarrami http://orcid.org/0000-0002-4034-1059

Michael Dinh http://orcid.org/0000-0002-0086-455X

Christine Lassen http://orcid.org/0000-0001-5552-2752

Hatem Alkhouri http://orcid.org/0000-0002-5569-704X

Lovana Daniel http://orcid.org/0000-0003-1241-6567

Brian Burns http://orcid.org/0000-0002-8794-0710

\section{REFERENCES}

1 WHO. Global report on drowning: preventing a leading killer. Geneva: World Health Organization, 2014.

2 Franklin RC, Peden AE, Hamilton EB, et al. The burden of unintentional drowning: global, regional and national estimates of mortality from the global burden of disease 2017 study. Inj Prev 2020;26:i83-95.

3 Lunetta P, Penttilä A, Sajantila A. Drowning in Finland: 'external cause' and 'injury' codes. Inj Prev 2002;8:342-4

4 Peden AE, Franklin RC, Mahony AJ, et al. Using a retrospective cross-sectional study to analyse unintentional fatal drowning in Australia: ICD-10 coding-based methodologies verses actual deaths. BMJ Open 2017;7:e019407.

5 Cenderadewi M, Franklin RC, Peden AE, et al. Fatal intentional drowning in Australia: a systematic literature review of rates and risk factors. PLoS One 2020;15:e0231861.

6 van Beeck EF, Branche CM, Szpilman D, et al. A new definition of drowning: towards documentation and prevention of a global public health problem. Bull World Health Organ 2005;83:853-6.

7 Mahony A, Barnsley P, Peden AE. A thirteen year national study of non-fatal drowning in Australia: data challenges, hidden impacts and social costs. Sydney: Royal Life Saving Society - Australia, 2017.

8 Manglick MP, Ross FI, Waugh M-C, et al. Neurocognitive outcomes in children following immersion: a long-term study. Arch Dis Child 2018;103:784-9.

9 Royal Life Saving Society - Australia. Royal life saving national drowning report 2019. Sydney: Royal Life Saving Society - Australia, 2019.

10 Barnsley PD, Peden AE, Scarr J. Calculating the economic burden of fatal drowning in Australia. J Safety Res 2018;67:57-63.

11 Cenderadewi M, Franklin RC, Peden AE, et al. Pattern of intentional drowning mortality: a total population retrospective cohort study in Australia, 2006-2014. BMC Public Health 2019;19:207.

12 Peden AE, Mahony AJ, Barnsley PD, et al. Understanding the full burden of drowning: a retrospective, cross-sectional analysis of fatal and non-fatal drowning in Australia. BMJ Open 2018;8:e024868.

13 Australian Institute of Health and Welfare. Leading causes of premature mortality in Australia fact sheet: accidental drowning. Canberra: Australian Institute of Health and Welfare, 2015.

14 Mitchell RJ, Curtis K, Holland AJ, et al. Acute costs and predictors of higher treatment costs for major paediatric trauma in New South Wales, Australia. J Paediatr Child Health 2013;49:557-63.

15 Mitchell RJ, Curtis K, Foster K. A 10-year review of child injury hospitalisations, health outcomes and treatment costs in Australia. Inj Prev 2018;24:344-50.

16 Zuckerbraun NS, Saladino RA. Pediatric drowning: current management strategies for immediate care. Clin Pediatr Emerg Med 2005;6:49-56.

17 Semple-Hess J, Campwala R. Pediatric submersion injuries: emergency care and resuscitation. Pediatr Emerg Med Pract 2014;11:1-21.

18 Wallis BA, Watt K, Franklin RC, et al. Drowning mortality and morbidity rates in children and adolescents $0-19$ yrs: a populationbased study in Queensland, Australia. PLoS One 2015;10:e0117948.

19 Matthews BL, Andrew E, Andronaco R, et al. Epidemiology of fatal and non-fatal drowning patients attended by paramedics in Victoria, Australia. Int J Inj Contr Saf Promot 2017;24:303-10.

20 Mitchell RJ, Williamson AM, Olivier J. Estimates of drowning morbidity and mortality adjusted for exposure to risk. Inj Prev 2010;16:261-6.

21 Centre for Health Record Linkage. Our services: centre for health record linkage, 2020. Available: https://www.cherel.org.au/ourservices [Accessed 6 Jul 2020].

22 Centre for Health Record Linkage. Technical details: centre for health record linkage, 2020. Available: https://www.cherel.org.au/howrecord-linkage-works/technical-details [Accessed 6 Jul 2020]

23 Centre for Health Record Linkage. How record linkage works: centre for health record linkage, 2020. Available: https://www.cherel.org.au/ how-record-linkage-works [Accessed 6 Jul 2020].

24 SAS Institute Inc. Sas analytics software and solutions. Cary, NC: SAS Institute Inc, 2019.

25 Australian Institute of Health and Welfare. Australian refined diagnosis-related groups $(A R-D R)$ data cubes. Canberra: Australian Institute of Health and Welfare, 2019. 
